\title{
Biópsia hepática por laparotomia paracostal em bovinos e búfalos
}

\author{
Paracostal liver biopsy in cattle and buffalo
}

\author{
Antonio Humberto Hamad Minervino' ${ }^{\mathrm{I}}$ Raimundo Alves Barrêto Júnior ${ }^{\mathrm{II}}$ \\ Frederico Augusto Mazzocca Lopes Rodrigues ${ }^{\mathrm{I}}$ Rodrigo Nogueira Fernandes Ferreira ${ }^{\mathrm{I}}$

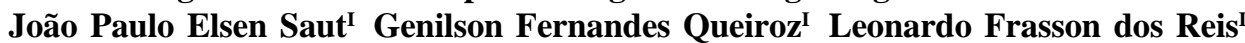 \\ Enrico Lippi Ortolani*
}

\section{RESUMO}

A técnica de biópsia hepática em ruminantes tem importante valor no diagnóstico clínico de doenças tóxicas e metabólicas, em especial nos desequilíbrios minerais. As técnicas mais comumente utilizadas restringem análises devido ao limitado volume de tecido obtido. No presente trabalho, avaliou-se o uso de uma técnica de biópsia hepática por laparotomia paracostal em bovinos e búfalos. Foram utilizados 10 bovinos e 10 búfalos hígidos. Os animais foram mantidos em estação, sedados com xilazina e infiltrados localmente com lidocaína e epinefrina. O acesso à cavidade abdominal foi realizado por meio de uma incisão dorso-ventral de $15 \mathrm{~cm}$ no flanco direito, iniciada ventralmente (cerca de 4-5cm) ao processo transverso da $2^{\underline{a}}$ ou $3^{\underline{a}}$ vértebra lombar e situada caudalmente (cerca de 4cm) e paralelamente à $13^{\underline{a}}$ costela, obtendo-se visualização do fígado. Foi então realizado pinçamento do bordo caudal do órgão com pinça Doyen para remoção de fragmento hepático (2 a 4g). Procedeu-se o fechamento da cavidade abdominal como de rotina. Foram analisados os parâmetros bioquímicos e hematológicos antes do procedimento (tempo zero) e após 24 horas, 48 horas, 5 dias e 10 dias após a biópsia. Todas as variáveis bioquímicas estudadas retornaram aos valores basais 5 e 10 dias após o procedimento nos bovinos e búfalos, respectivamente. O tempo médio de cirurgia por animal foi de 25 minutos. A biópsia hepática por laparotomia paracostal demonstrou ser uma técnica eficaz e de baixo risco à saúde dos animais, permitindo a coleta de suficiente quantidade de tecido hepática para realização de múltiplas análises.

Palavras-chave: fígado, bovinos, bufalinos, laparotomia, hematologia, bioquímica.

\section{ABSTRACT}

Liver biopsy in ruminants is an important technique for clinical diagnosis of toxic and metabolic diseases, especially mineral disorders. The most frequent procedures used so far results in an small amount of liver and not enough for multiple tests. The present study aims to evaluate the efficacy of paracostal laparotomy liver biopsy technique in cattle and buffalo and to follow possible changes on biochemical and hematological profiles caused by the surgery. Ten cattle and ten buffalo were used in this study. The animals were kept on their normal position and sedated with xilazin and locally infiltrated with lidocain and epinefrin. The access to abdominal cavity was carried through a right paracoastal laparotomy, ventrally to the transverse process of $2^{\text {nd }}$ or $3^{\text {rd }}$ lombar vertebra. A small portion of the hepatic caudal lobe (2 to $4 \mathrm{~g}$ ) was clinched by a Doyen clamp and removed. The abdominal cavity was closed as normally routine. Blood samples were taken to biochemical tests and hemogram before the procedure, and 24h, 48h, 5 and 10 days later. The technique was practical and easy to be performed (took approximately 25 minutes). The technique described here was efficient and offers low risk to animal health and provided enough amount of liver tissue for multiple tests.

Key words: liver, cattle, buffaloes, laparotomy, hematology, biochemistry.

\section{INTRODUÇÃO}

O metabolismo hepático tem destacada importância nos estudos dos desequilíbrios minerais e intoxicações por várias substâncias. Isso se deve ao

IFaculdade de Medicina Veterinária e Zootecnia (FMVZ), Universidade de São Paulo (USP), Av. Prof. Orlando Marques de Paiva, 87, Cidade Universitária, 05508-900, São Paulo, SP, Brasil. E-mail: ortolani@usp.br. *Autor para correspondência.

"Universidade Federal Rural do Semi-Árido (UFERSA), Mossoró, RN, Brasil. 
fato de que o fígado armazena vários elementos como o cobre, cobalto e chumbo, sendo que as concentrações minerais podem se alterar no fígado antes de serem percebidas alterações dos valores sangüíneos (UNDERWOOD \& SUTLE, 1999). Além dos teores minerais no fígado, recentemente, vem se destacando no estudo do metabolismo mineral, especialmente em relação ao cobre e zinco, a avaliação da metalotioneina hepática (LOPEZ-ALONSO et al., 2005). Desse modo, a técnica de biópsia hepática em ruminantes tem importante valor, tanto no diagnóstico clínico de deficiências minerais, como na comprovação de determinadas intoxicações (BARROS et al., 2006; ORTOLANI, 2008).

Dentre as técnicas de biópsia utilizadas em medicina veterinária, destacam-se a biópsia por laparoscopia, utilizada principalmente em equinos. Esse é um método pouco invasivo e eficaz especialmente para análises histopatológicas (SILVA et al., 2002). A biópsia laparoscópica por cauterização, utilizada em cães, também apresenta resultados satisfatórios, sem provocar alterações hematológicas e bioquímicas (FANTINATTI et al., 2003). Ambas as técnicas descritas têm como desvantagem o elevado custo dos equipamentos e a dificuldade de execução a campo.

Em ruminantes, as biópsias realizadas para análises histopatológicas necessitam de pequena quantidade de amostra. BRAGA et al. (1985) recomendam a utilização da agulha de Menghini já que a mesma apresenta as vantagens de ser rápida, não necessitar de anestesia e poder ser realizada a campo. Em bovinos, WITZEL et al. (1975) descrevem como uma nova técnica de biópsia hepática a remoção parcial de um lobo do fígado, com retirada de $25 \mathrm{~g}$ de tecido. A técnica por aspiração, realizada com o uso de um trocater específico é comumente utilizada em bovinos e também apresenta resultados satisfatórios em relação à recuperação do animal após o procedimento (AMORIN et al., 2003; BARROS et al., 2006).

Dentre as técnicas supramencionadas, com exceção da lobectomia parcial, a principal limitação observada está relacionada à quantidade de tecido hepático obtido, que pode ser insuficiente para a realização de múltiplas análises (MILES et al., 2001; LOPEZ-ALONSO et al., 2005). Desse modo, no presente trabalho objetivou-se avaliar a exequibilidade e eficácia da técnica de biópsia hepática por laparotomia paracostal em bovinos e búfalos e verificar as possíveis alterações na bioquímica sérica e no hemograma desses animais após a técnica cirúrgica.

\section{MATERIAL E MÉTODOS}

Foram utilizados 20 animais (10 bovinos e 10 búfalos), machos, hígidos, procedentes de fazendas do Estado de São Paulo, com cerca de doze meses de idade e média de 180 e $200 \mathrm{~kg}$ de peso vivo para bovinos e búfalos, respectivamente. $\mathrm{O}$ acesso à cavidade abdominal foi realizado na fossa paralombar direita, por meio de uma incisão dorso-ventral de $15 \mathrm{~cm}$ iniciada ventralmente (cerca de $4-5 \mathrm{~cm}$ ) ao processo transverso da $2^{\underline{a}}$ ou $3^{\underline{a}}$ vértebra lombar e situada caudalmente (cerca de $4 \mathrm{~cm}$ ) e paralelamente à $13^{\text {a }}$ costela, obtendo-se assim excelente visualização do fígado. A anestesia foi realizada com cloridrato de xilazina $^{\mathrm{a}}$ a $2,3 \%$ na dose de $0,5 \mathrm{~mL}$ para $100 \mathrm{~kg}$ de peso vivo, com a finalidade de tranqüilização e anestesia local de pele e musculatura por infiltração de cloridrato de lidocaína associada à epinefrina ${ }^{\mathrm{b}}$ no local da incisão. Foram seccionados a pele e os músculos oblíquos abdominais externo e interno, seguida da secção com tesoura do músculo transverso e do peritônio, o que permitiu exposição do fígado. Foi realizado pinçamento do lobo caudal hepático, utilizando-se uma pinça de Doyen que permaneceu por, no mínimo, cinco minutos no local, mesmo após a secção e retirada do fragmento. Na figura 1, ilustram-se alguns dos procedimentos cirúrgicos realizados.

A cavidade abdominal foi fechada com padrão de sutura simples continua com fio catgute $n^{0}$ 2, aproximando-se peritônio e músculo transverso. Em seguida, efetuou-se a sutura dos músculos oblíquos abdominais e fáscia do subcutâneo com o mesmo padrão e fio de sutura descritos acima. A pele foi aproximada em padrão de sutura de Wolf com fio de náilon 0,6mm. Com o objetivo de evitar infecção e/ou inflamação aguda no pós-operatório, logo depois do procedimento, foi administrada uma associação de antibiótico e antiinflamatório de longa ação na dose de $1 \mathrm{~mL}$ para cada $10 \mathrm{~kg}$ de peso vivo em todos os animais (oxitetracilcina $20 \%$ e diclofenaco sódico 0,5\%c).

Antes do inicio da biópsia, tempo zero (T0) e após 24 horas (T24h), 48 horas (T48h), 5 dias (T5d) e 10 dias (T10d) da realização da técnica, foram coletadas amostras de sangue obtidas por meio de venipunctura da jugular externa, utilizando-se tubos de coleta a vácuo. Para as determinações bioquímicas da proteína total, albumina e atividade enzimática da gamaglutamiltransferase (GGT), aspartatoamino-transferase (AST) e creatina quinase (CK), o sangue foi coletado em tubos a vácuo sem anticoagulante para a obtenção do soro, que foi transferido para tubos plásticos e armazenado em freezer a $20^{\circ} \mathrm{C}$ negativos. As determinações bioquímicas foram realizadas no analisador automático ${ }^{\mathrm{d}}$ utilizando-se kits comerciais. Os resultados das 


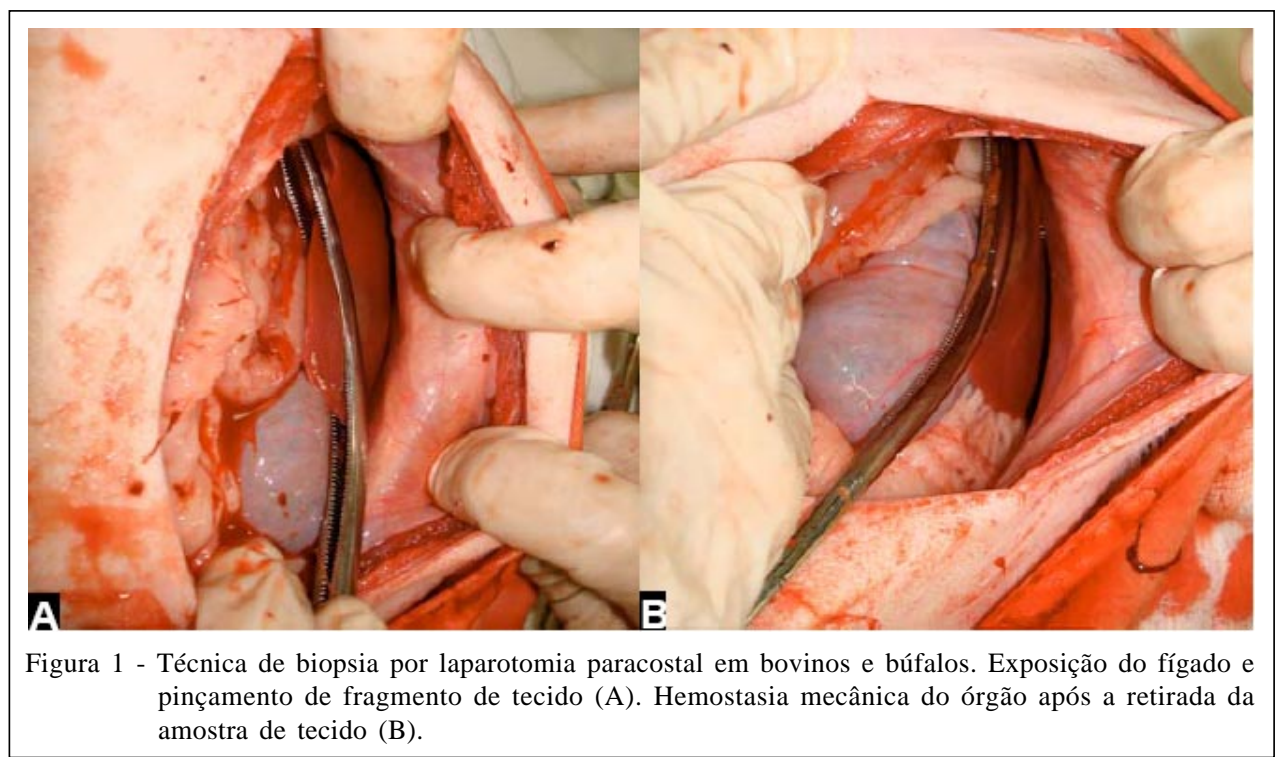

atividades das enzimas foram determinados em $30^{\circ} \mathrm{C}$ segundo recomendações de KANEKO et al. (1997).

Para as análises hematológicas, realizou-se coleta de sangue com tubos a vácuo com anticoagulante (EDTA) que foram mantidos em refrigeração $\left(4^{\circ} \mathrm{C}\right)$ até a análise. O volume globular (VG) foi determinado pela técnica de microcentrifugação em tubo capilar (FELDMAN et al., 2000). A contagem total de hemácias, teor de hemoglobina, contagem total de leucócitos, volume corpuscular médio (VCM), hemoglobina corpuscular média (HCM) e a concentração de hemoglobina corpuscular média (CHCM) foram determinados com a utilização de contador automático de célulase ${ }^{e}$.

A análise estatística dos resultados foi realizada com auxílio de software estatístico (MINITAB, 2000). Inicialmente, utilizou-se o teste de KolgomorovSmirvov para determinação da distribuição dos dados. Para análise das variáveis paramétricas, foi aplicado o teste $\mathrm{T}$ pareado para amostras dependentes e, nas amostras não paramétricas, optou-se pelo teste de Wilcoxon, em ambos os casos utilizando-se intervalo de confiança de $95 \%$.

\section{RESULTADOS E DISCUSSÃO}

A técnica de biópsia empregada mostrou aplicabilidade prática, pois o procedimento cirúrgico foi realizado com o animal em estação, em um período de cerca de 25 minutos. O fragmento hepático obtido teve peso fresco, aproximado, de 2 a 4 gramas, permitindo a realização de várias análises. Na tabela 1 , apresentam-se os resultados da bioquímica sérica em bovinos e búfalos nos diferentes tempos de coleta, enquanto na tabela 2, estão os resultados do hemograma desses animais nos supracitados momentos. Não ocorreram alterações nas variáveis do perfil bioquímico em ambas as espécies no T10d, demonstrando que a técnica é segura e que, após esse período, os animais estão plenamente recuperados. Nos bovinos, ocorreu uma mínima redução da proteína total nos tempos T24h e T48h. Nesses mesmos animais, houve redução da albumina sérica no T48h. Provavelmente, esse foi o fator responsável pela manutenção da redução da proteína total nesse tempo.

A atividade da AST apresentou incremento no T24h e T48h, nos bovinos e no T24h, T48h e T5d nos búfalos. Essa elevação ocorreu concomitantemente ao aumento da atividade da CK, o que pôde ser verificado pelas altas correlações positivas entre as duas enzimas detectadas nos bovinos $\left(\mathrm{r}^{2}=0,615\right)$ e nos búfalos $\left(r^{2}=0,504\right)$. Não foram observadas alterações na atividade da GGT em nenhum dos momentos avaliados em ambas as espécies. Esse último resultado sugere que as lesões hepáticas durante a biópsia no bordo distal do fígado não foram significativamente grandes para serem notificadas pelo aumento da atividade da GGT, a qual se eleva toda vez que ocorrer agressão ou multiplicação de células de canalículos biliares, com baixa presença nessa região biopsiada. Embora a biopsia possa ter provocado extravasamento de AST para a corrente sanguínea (AMORIN et al., 2003), acredita-se que maior percentual de atividade dessa enzima, baseado nos resultados de CK, seja proveniente da lesão muscular decorrente do processo cirúrgico. A elevação da CK ocorreu nos bovinos e búfalos no T24h, permanecendo com valores elevados até o T48h, em bovinos e até o T5d, nos búfalos. 
Tabela 1 - Bioquímica sérica em bovinos e búfalos antes a após técnica de biópsia hepática.

\begin{tabular}{|c|c|c|c|c|c|}
\hline & Т 0 & $\mathrm{~T} 24 \mathrm{~h}$ & $\mathrm{~T} 48 \mathrm{~h}$ & T 5d & T 10d \\
\hline Bovinos & Média \pm DP & Média \pm DP & Média \pm DP & Média \pm DP & Média \pm DP \\
\hline Proteína total $\left(\mathrm{g} \mathrm{dL}^{-1}\right)$ & $7,1 \pm 0,5$ & $6,8^{*} \pm 0,6$ & $6,5^{* *} \pm 0,5$ & $6,8 \pm 0,7$ & $7,1 \pm 0,5$ \\
\hline Albumina ( $\mathrm{g} \mathrm{dL}^{-1}$ ) & $2,7 \pm 0,2$ & $2,7 \pm 0,1$ & $2,6^{*} \pm 0,2$ & $2,6 \pm 0,2$ & $2,8 \pm 0,1$ \\
\hline GGT $\left(\mathrm{U} \mathrm{L}^{-1}\right)$ & $21 \pm 18,5$ & $22 \pm 17,5$ & $21 \pm 16,3$ & $18 \pm 17,5$ & $22 \pm 17,1$ \\
\hline $\operatorname{AST}\left(\mathrm{U} \mathrm{L}^{-1}\right)$ & $62 \pm 15,2$ & $108^{* *} \pm 27,1$ & $99 * * \pm 25,2$ & $71 \pm 26,3$ & $59 \pm 22,6$ \\
\hline $\begin{array}{l}\left.\text { CK (U L L }{ }^{-1}\right) \text { (Mediana) } \\
\text { Búfalos }\end{array}$ & 121 & $967^{* *}$ & $487 * *$ & 91 & 93 \\
\hline Proteína total ( $\mathrm{g} \mathrm{dL}^{-1}$ ) & $6,5 \pm 0,4$ & $6,4 \pm 0,5$ & $6,4 \pm 0,4$ & $6,9 \pm 0,7$ & $7,0 \pm 0,6$ \\
\hline Albumina (g dL ${ }^{-1}$ ) & $2,8 \pm 0,1$ & $2,7 \pm 0,1$ & $2,7 \pm 0,3$ & $2,8 \pm 0,2$ & $2,9 \pm 0,2$ \\
\hline GGT $\left(\mathrm{U} \mathrm{L}^{-1}\right)$ & $18 \pm 6,9$ & $17 \pm 5,8$ & $16 \pm 6,2$ & $16 \pm 5,5$ & $17 \pm 7,4$ \\
\hline $\operatorname{AST}\left(\mathrm{U} \mathrm{L}^{-1}\right)$ & $120 \pm 31,9$ & $171^{* *} \pm 49,5$ & $159 * \pm 48,6$ & $168 * * \pm 34,3$ & $135 \pm 34,7$ \\
\hline CK (U L $\left.{ }^{-1}\right)$ (Mediana) & 84 & $3356 * *$ & $1256^{* *}$ & $427 *$ & 105 \\
\hline
\end{tabular}

* Diferença significativa em relação ao tempo zero $(\mathrm{P}<0,05)$. ** Altamente significativo $(\mathrm{P}<0,01)$.

O VG esteve elevado, no T24h, em ambas as espécies, entretanto permaneceu dentro dos valores de referência (LATIMER et al., 2003) em todos os momentos de coleta. Esse aumento do VG logo após a biópsia foi provavelmente devido à elevação da quantidade de hemácias que ocorreu no T24h, que deve ser decorrente da liberação de células para a corrente sanguínea, sendo, possivelmente, uma policitemia transitória por contração esplênica (FELDMAN et al., 2000).

Os teores de hemoglobina se elevaram no T24h em bovinos e búfalos, ocorrendo redução dos valores basais já no T48h, provavelmente, devido à restauração do volume sanguíneo através da passagem de fluido intersticial, que se inicia 2 a 3 horas e persiste até 72 horas após a hemorragia (LATIMER et al., 2003). Ocorreu uma nova elevação dos valores de hemoglobina no T10d nos bovinos e T5d nos búfalos. Os motivos para tal alteração são desconhecidos. A quantidade de leucócitos se elevou no T24h e T48h em ambas as espécies, porém não ultrapassou os valores de referência, retornando aos valores basais já no 5o dia após a biópsia.

Comparando-se os resultados hematológicos do T5d com os valores obtidos 96 horas após a técnica de biópsia descrita por AMORIN et al. (2003), observaram-se resultados semelhantes. Em ambos os casos, a técnica cirúrgica utilizada não provocou

Tabela 2 - Hemograma de bovinos e búfalos antes a após técnica de biópsia hepática.

\begin{tabular}{|c|c|c|c|c|c|}
\hline & Т 0 & Т 24 & Т 48 & T 5D & T 10D \\
\hline Bovinos & Média \pm DP & Média \pm DP & Média \pm DP & Média \pm DP & Média \pm DP \\
\hline Volume globular (\%) & $26,2 \pm 1,4$ & $30,3^{* *} \pm 1,0$ & $27,6 \pm 1,6$ & $28,2 \pm 3,3$ & $27,2 \pm 1,1$ \\
\hline Hemácias (x $10^{6} \mu \mathrm{L}^{-1}$ ) & $7,3 \pm 0,8$ & $8,3^{* *} \pm 0,7$ & $7,3 \pm 1,0$ & $7,4 \pm 1,1$ & $7,5 \pm 1,0$ \\
\hline Hemoglobina ( $\mathrm{g} \mathrm{dL}^{-1}$ ) & $8,1 \pm 0,5$ & $9,1^{* *} \pm 0,5$ & $8,2 \pm 0,9$ & $8,8 \pm 0,8$ & $9,1^{*} \pm 0,5$ \\
\hline Leucócitos (x $\left.10^{3} \mu \mathrm{L}^{-1}\right)$ & $9,3 \pm 3,2$ & $12,2 * * \pm 3,5$ & $12,1^{* *} \pm 3,5$ & $10,5 \pm 1,7$ & $11,6 \pm 2,0$ \\
\hline VCM (fL) & $36,4 \pm 3,4$ & $36,6 \pm 3,2$ & $36,9 \pm 3,6$ & $39,4^{*} \pm 6,2$ & $39,7^{*} \pm 6,2$ \\
\hline HCM (pg) & $11,3 \pm 0,9$ & $11,1 \pm 0,9$ & $11,3 \pm 0,9$ & $12,1^{*} \pm 1,5$ & $12,2 * \pm 1,2$ \\
\hline $\begin{array}{l}\left.\text { CHCM (g dL }{ }^{-1}\right) \\
\text { Búfalos }\end{array}$ & $31,1 \pm 0,9$ & $30,5 \pm 1,3$ & $30,8 \pm 1,7$ & $30,7 \pm 1,6$ & $31,1 \pm 2,1$ \\
\hline Volume globular (\%) & $29,1 \pm 3,7$ & $32,3^{* *} \pm 3,6$ & $29,8 \pm 2,2$ & $32,2 \pm 3,9$ & $31,8 \pm 4,6$ \\
\hline Hemácias (x $10^{6} \mu L^{-1}$ ) & $7,3 \pm 0,9$ & $8,1^{* *} \pm 0,8$ & $7,4 \pm 0,5$ & $7,3 \pm 0,7$ & $7,4 \pm 0,9$ \\
\hline Hemoglobina ( $\mathrm{g} \mathrm{dL}^{-1}$ ) & $9,0 \pm 1,0$ & $9,9 * * \pm 0,9$ & $9,3 \pm 0,5$ & $10,3^{*} \pm 1,0$ & $9,5 \pm 1,4$ \\
\hline Leucócitos (x $\left.10^{3} \mu \mathrm{L}^{-1}\right)$ & $10,1 \pm 3,8$ & $11,5^{*} \pm 3,6$ & $11,9^{* *} \pm 3,8$ & $10,3 \pm 2,3$ & $10,6 \pm 2,9$ \\
\hline VCM (fL) & $39,6 \pm 1,7$ & $39,9 \pm 1,7$ & $40,3 \pm 1,5$ & $44,1^{* *} \pm 5,4$ & $43,0 * \pm 5,5$ \\
\hline HCM (pg) & $12,3 \pm 0,6$ & $12,2 \pm 0,5$ & $12,6 \pm 0,4$ & $14,0^{* *} \pm 1,3$ & $12,8 \pm 1,9$ \\
\hline CHCM $\left(\mathrm{g} \mathrm{dL}^{-1}\right)$ & $30,9 \pm 0,7$ & $30,6 \pm 1,0$ & $31,2 \pm 0,9$ & $32,0 \pm 1,5$ & $29,9 \pm 1,2$ \\
\hline
\end{tabular}

* Diferença significativa em relação ao tempo zero $(\mathrm{P}<0,05)$. ** Altamente significativo $(\mathrm{P}<0,01)$.

Ciência Rural, v.39, n.3, mai-jun, 2009. 
alteração dos valores normais dos principais constituintes do hemograma.

No estudo de WITZEL et al. (1975), apesar da recuperação plena dos animais, as alterações na função do órgão não foram avaliadas. Com a crescente preocupação com o bem-estar animal, um procedimento como esse, que remove parcialmente um lobo hepático, deve ser evitado.

Em comparação com a técnica descrita por AMORIN et al. (2003), o procedimento estudado apresenta a vantagem de permitir a colheita de um maior fragmento hepático, necessário, principalmente, em pesquisas que visam determinar a concentração mineral e de metaloenzimas, como a metalotioneína hepática (MILES et al., 2001; LOPEZ-ALONSO et al., 2005).

Em relação às técnicas descritas por SILVA et al. (2002) e FANTINATTI et al. (2003), além de apresentarem a desvantagem da limitada quantidade de tecido retirado, são de difícil exeqüibilidade em bovinos, em especial, quando se deseja realizar o procedimento no local de criação, pois ambas as técnicas necessitam de equipamentos caros e de uso incomum do veterinário buiatra.

\section{CONCLUSÕES}

Pode-se concluir que a biópsia hepática por laparotomia mostrou-se eficaz e sem risco à saúde dos animais, permitindo a colheita de quantidade suficiente de amostra para a realização de múltiplos testes. A técnica é indicada para a realização de pesquisas que objetivam analisar diferentes variáveis hepáticas, também podendo ser utilizada na confirmação de diagnóstico clínico de deficiências ou intoxicações minerais.

\section{AGRADECIMENTOS}

Pesquisa financiada pela Fundação de Amparo à Pesquisa do Estado de São Paulo (FAPESP) (Bolsa de mestrado nº 2005/01144-0; auxílio pesquisa nº2005/03204-0).

Os autores são gratos às técnicas Clara S. Mori, Samantha Ive Miyashiro e Marly Elizabete F. de Castro pelo auxílio com as análises laboratoriais.

\section{FONTES DE AQUISIÇÃO}

\author{
a - Anasedan ${ }^{\circledR}$ - Vetbrands ${ }^{\circledR}$ Saúde Animal \\ b - Anestésico local Pearson ${ }^{\circledR}$ \\ c - Reverin ${ }^{\circledR}$ L.A. Plus. Intervet ${ }^{\circledR}$ Brasil \\ d - Analisador automático modelo Liasys, AMS ${ }^{\circledR}$, Roma, Itália. \\ e - ABC ${ }^{\circledR}$ - Animal Blood Counter, HORIBA ABX, São Paulo, \\ Brasil.
}

\section{COMITÊ DE BIOÉTICA}

Este estudo foi aprovado pela comissão de bioética da Faculdade de Medicina Veterinária e Zootecnia da Universidade de São Paulo, em 25 de outubro de 2005. Protocolo número $746 / 2005$.

\section{REFERÊNCIAS}

AMORIN, R.M. et al. Bioquímica sérica e hemograma de bovinos antes e após a técnica de biópsia hepática. Ciência Rural, v.33, n.5, p.519-523, 2003.

BARROS, C.S.L. et al. Biópsia hepática no diagnóstico da intoxicação por Senecio brasiliensis (Asteraceae) em bovinos. Pesquisa Veterinária Brasileira, v.27, n.1, p.53-60, 2006. Disponível em: http://www.scielo.br/scielo.php?script=sci_arttext\&pid=S0100736X2007000100010\&lng=en\&nrm=iso\&tlng=pt. Doi: 10.1590/ S0100-736X2007000100010.

BRAGA, M.R. et al. Biópsia hepática em bovinos: proposta de nova técnica. Revista do Centro de Ciências Rurais, v.15, n.1, p.79-88, 1985.

FANTINATTI, A.P. et al. Laparoscopy hepatic biopsy through cauterization. Ciência Rural, v.33, n.4, p.703707, 2003. Disponível em: http://www.scielo.br/ scielo.ph p s c ri pt=s ci_art text \& pid=s 0103 $84782003000400019 \& \operatorname{lng}=$ en $\& n r m=i s o \& t \operatorname{lng}=$ en. Doi: 10.1590/S0103-84782003000400019.

FELDMAN, B.F. et al. Schalm's veterinary hematology, 5.ed. Philadelphia: Lippincott Williams \& Wilkins, 2000. $1.344 \mathrm{p}$.

KANEKO, J.J. et al. Clinical biochemistry of domestic animals. 5.ed. San Diego: Academic, 1997. 932p.

LATIMER, K.S. et al. Duncan and Prasse's veterinary laboratory medicine: clinical pathology. 4.ed. Ames: Iowa State, 2003. 468p.

LÓPEZ-ALONSO, M. et al. The role of metallothionein and zinc in hepatic copper accumulation in cattle. Veterinary Journal, v.169, p.262-267, 2005. Disponível em: http:// w w w. s c i e $n$ c e d i r e c t c c m / science?_ob=ArticleURL\&_udi=B6WXN-4C1FCJR$3 \&$ \&_u s e r $=687358 \&$ r d o c $=1 \&$ \& m t $=\&$

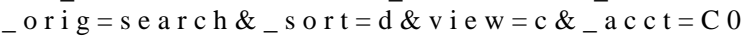
$00037899 \&_{\text {_ version }}=1 \&_{\text {_ u r l Version }}=0 \&_{-}$u serid $=687358 \& m d 5=8986557291736 b 29181$ bbf839cc7e32a. Doi: 10.1016/j.tvjl.2004.01.019.

MILES, H.M. et al. Analysis of minerals for animal nutrition research. 3.ed. Gainesville: University of Florida, 2001. 117p.

MINITAB - The student edition of MINITAB Statistical software adapted for education. Release 13.0. User's manual. New York: Addison-Wesley, 2000. CD-ROM.

ORTOLANI, E.L. Intoxicação por cobre, selênio, zinco, cloreto e sódio. In: SPINOSA, E.S. et al. (Org.). Toxicologia aplicada à medicina veterinária. São Paulo: Manole, 2008.

SILVA, L.C.L.C. et al. Técnica de biópsia hepática em eqüino por laparoscopia. Ciência Rural, v.32, n.3, p.459-465, 2002

UNDERWOOD, E.J.; SUTTLE, N.F. The mineral nutrition of livestock. 3.ed. Wallingford: Cabi Publishing, 1999. 614p.

WITZEL D.A. et al. Partial lobectomy of bovine liver: a new biopsy technique. Cornell Veterinarian, v.65, n.1, p.112119, 1975. 